ARXIU D'ETNOGRAFIA DE CATALUNYA, N² 2,1983

\title{
SANTUARIOS MARIANOS DE LA DIÓCESIS DE TARRAGONA: APROXIMACIÓN GEOGRÁFICA
}

Diego López Bonillo 


\section{INTRODUCCION}

Uno de los aspectos a tener en cuenta en el estudio de los santuarios marianos, considerados como centros de religiosidad popular, es el denominado ecológico, es decir, las características generales del entorno en el que se encuentran insertos, contempladas desde un punto de vista geográfico. En este sentido hay que tener en cuenta varios aspectos susceptibles de esquematización, como situación en el contexto general del área estudiada, caracteristicas fisicas del emplazamiento, relaciones con el hábitat, etc.

Desde este punto de vista, los santuarios marianos del obispado de Tarragona, aunque están perfectamente singularizados en su en. torno, presentan una serie de constantes en relación con el espacio geográfico de que forman parte, que permite una cierta sistematización dentro del conjunto.

En el presente trabajo se intentan poner en evidencia los puntos de coincidencia en el conjunto y los rasgos que singularizan cada uno de los centros estudiados. Hay que tener en cuenta, no obstante, que consiste en una aproximación, siendo susceptible de una investigación más exhaustiva todos y cada uno de los puntos tratados.

\section{ÁmBITO DE ESTUdio}

El arzobispado de Tarragona abarca, en su dimensión actual, la totalidad de los territorios comprendidos en las comarcas del Tarragonès, Alt Camp, Conca de Barberà y Baix Camp (excepto una pequeña porción del extremo sur), así como fragmentos del Priorat, Garrigues, Urgell, Segarra y Baix Penedès. Los limites actuales son el re- 
sultado de la remodelación llevada a cabo en 1957, año en que adquirió territorios pertenecientes hasta entonces a las diócesis de Vic y Barcelona. El presente estudio se ha realizado en base a los límites anteriores a dicha remodelación.

\section{Caracteres físicos}

El relieve presenta una cierta simplicidad, dentro de la diversidad de formaciones. Está recorrido el territorio de NE a SW por la cordillera Prelitoral, que presenta varias unidades:

Bloque del Gaià, en el curso superior y medio de este río, sirve de separación con la comarca del Penedès; una estribación sigue la dirección sur, descendiendo hasta la costa.

Sierra de Miramar, limite natural entre el Camp de Tarragona y la Conca de Barberà, enlaza el bloque del Gaià con las sierras de Prades.

Montañas de Prades. Extenso bloque con las alturas mayores del territorio considerado (Baltasana, $1201 \mathrm{~m}$ ), se eleva bruscamente sobre el Camp, presentando altiplanos por encima de los 800 metros: surcado por valles profundos de los ríos que tienen su cabecera en este sector, actúa como centro dispersor de aguas.

- Sierra del Montsant (1166 m.). De disposición longitudinal entre los valles de los rios Siurana y Montsant, con paredes verticales a ambas vertientes, contrasta violentamente con los relieves suaves del Priorat. Al norte y en dirección paralela, la sierra de La Llena marca el limite con las comarcas de la Depresión Central.

La llanura del Camp de Tarragona; triangular, abierta hacia el mar, desciende suavemente hacia la costa y se halla enmarcada perfectamente por las formaciones de la cordillera Prelitoral.

- La Conca de Barberà; cubeta alargada y separada del resto de la Depresión Central por un reborde montañoso que desde el fondo de la depresión adquiere una cierta entidad.

El clima es tipicamente mediterráneo, con las variaciones que imprime el relieve como factor modificante.

\section{Santuarios}

En el ámbito considerado, se encuentran los centros marianos que figuran en el cuadro 1.

Cuadro $n^{\circ}$ 1.- Santuarios marianos en la diócesis de Tarragona (1).

\section{Siglo \\ Ermita o}

Comarca

Altura
$\frac{X I}{X I I}$

$\left(\frac{10}{10}\right.$

Tallat

Puigcerver

El Cami

Siurana

Els Prats ${ }^{(3)}$

La Bovera

La Serra

Passanant

El Burgar

Paret Delgada

El Claustre

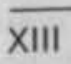

XIII

Montsan
La Roca

La Jonquera

Montornès

La Pineda

Montgoi

\section{XIV}

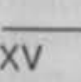

La Riera

El Lledó

$\mathrm{XV}$

XVI

Barrulles

Valldossera

Loreto

Misericòrdia

El Roser

El Roser

El Roser

XVII

XVIII Loreto

La Gràcia

El Remei ${ }^{(4)}$
La Consolació

Berà

Loreto

Pobla de MafumetTarragonès

Alforja |Baix Camp

Baix Camp

Cambrils

Priorat

\section{Siurana}

Conca Barberà

Guimerà

Montblanc

Urgell

Passanant

Conca Barberà

Reus

Conca Barberà

Selva del Camp

Baix Camp

Vallbo. Monges

Baix Camp

Albarca

Montroig

Urgel

Pobla Cérvoles

Priorat

Pobla Montornès Tarragonès

Baix Camp

Les Garrigues

Vilaverd

Tarragonès

Albiol

Borges del Camp

Conca Barberà

\begin{tabular}{l|l} 
Baix Camp & 780
\end{tabular}

Valls

Baix Camp

Conca Barberà $\quad 470$

Capafonts

Baix Camp 740

\begin{tabular}{l|l|l}
\hline Montagut(Querol) & Alt Camp & 610
\end{tabular}

Tarragonès

Baix Camp

Baix Camp

Alt Camp

Alt Camp

Alt Camp

Alt Camp

Priorat

Tarragonès

Priorat

Roda de Berà

Ulldemolins 
Son un total de treinta y seis santuarios y ermitas de caracteristicas muy diversas, tanto en lo que respecta a los factores que los individualizan como a los caracteres geográficos en el que están ubicados.

\section{Distribución espacial}

La primera consideración a tener en cuenta es la distribución en el ámbito del territorio estudiado. A este respecto, es digno de seña. lar cómo la concentración en unos sectores contrasta con la existen. cia de espacios vacios en otros (figura 1). Son particularmente abudantes en el sector centro y occidental, dándose la mayor densidad en la linea imaginaria Reus-Valls.

Digno de resaltar, asimismo, es la abundancia de santuarios en el sector montañoso occidental; las montañas que cierran el Camp por este lado y que se prolongan hasta el Montsant y sierra de La Llena, se encuentran prácticamente rodeadas por un cinturón de eremitorios que, completados por otros en el interior, dotan a este territorio de una elevada densidad de centros marianos.

En contraste con esta abundancia, se registra el vacío de los territorios situados al Norte y Este de la diócesis; este vacio está roto únicamente por los cuatro del extremo septentrional de la Conca de Barberà y Valldossera en el límite oriental con la comarca del Penedès.

Las razones de esta irregular distribución no son indudablemen. te de indole geográfica, puesto que dentro de la variedad de condicio. nes físicas del territorio en cuestión, éstas no son lo suficientemente contrastadas para explicar la ausencia de centros en ciertos territo. rios mientras abundan en otros. La densidad de población en el sec. tor Ilano del Camp de Tarragona podría explicar la abundancia de centros y éste es un factor a tener en cuenta; pero no explica por sí mismo el fenómeno en cuestión. En efecto, en el resto de la diócesis se dan densidades, pero en ningún caso puede establecerse una co. rrelación entre ambas variables. Pensamos que las razones de la dis. tribución espacial hay que buscarlas en las circunstancias históricas existentes en la época en que fueron erigidos los centros estudiados.

De la observación del cuadro 1 se deduce que los centros más antiguos se remontan al siglo XII, con la excepción de EI Tallat y tal vez de Els Prats, cuyos origenes parecen situarse en el siglo anterior. En esta época la divisoria entre la Catalunya cristiana y la demarcación ocupada por los musulmanes se situaba en el Gaià, precisamen. 


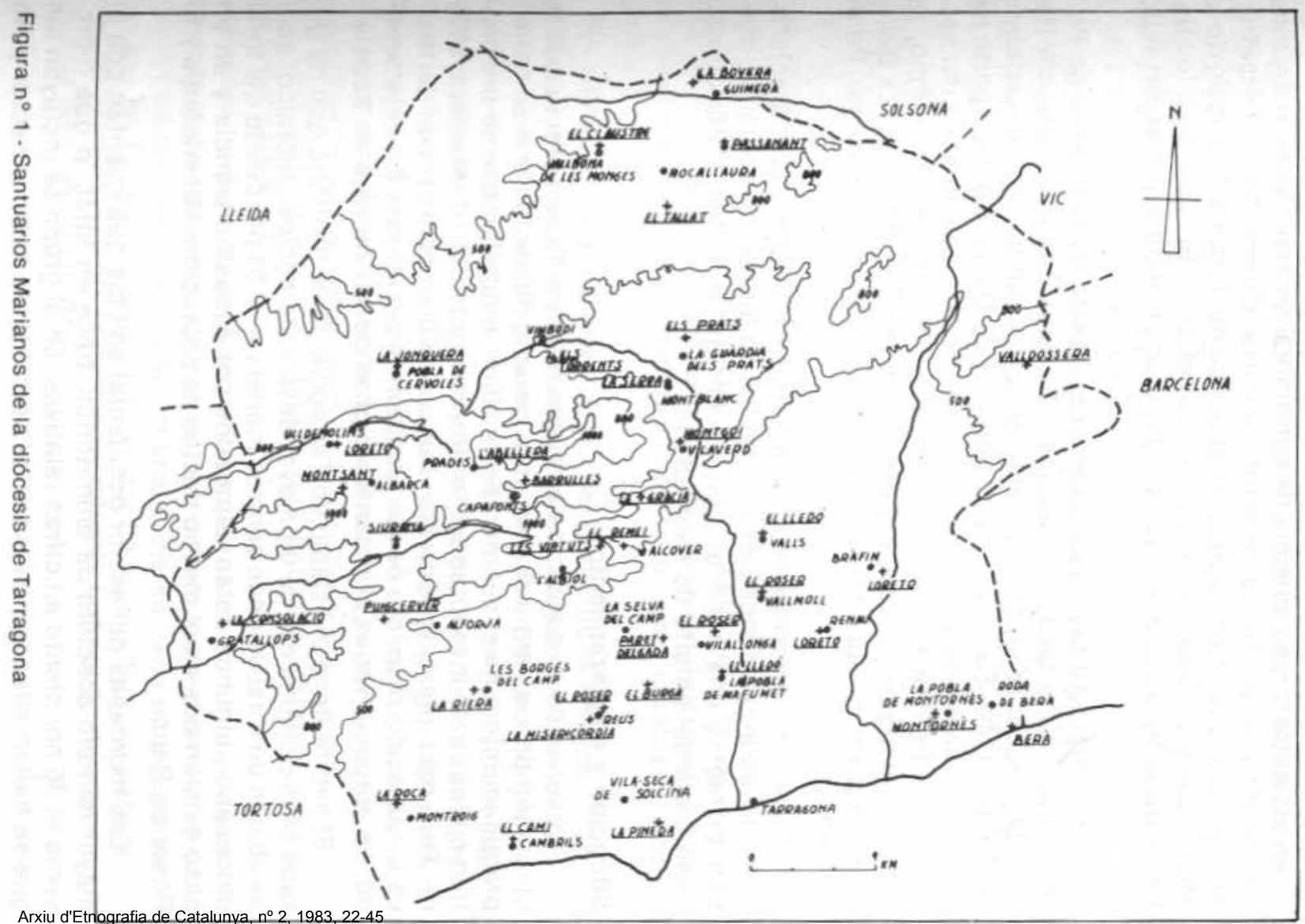

ISSN: 0212-0372. EISSN: 2014-3885

http://antropologia.urv.cat/revistarxiu 
te en el sector cuya ausencia de santuarios es manifiesta; esta zona se encontraba sembrada de fortificaciones -Querol, Santa Perpetua, etc.- al igual que otros lugares de la Conca de Barberà -Montbrió de la Marca, Biure de Gaià, Savallà del Comptat, etc.-- El curso bajo fue tierra de nadie hasta que se inició la repoblación lenta a finales del siglo XI.

En el siglo XII tuvo lugar la reconquista de las montañas de Prades, cuyo último bastión musulmán, Siurana, pasó a manos de Ramón Berenguer IV en 1153. En esta época se fundan los monasterios de Poblet (1149), Santes Creus (1159) y Scala Dei (1163). Es a partir de entonces cuando se edifican la mayor parte de los santuarios del sector montañoso de Prades y sus estribaciones. Al mismo tiempo, se establecen algunos en los lugares ya repoblados del Norte -La Bovera, Passanant, El Claustre-o de antigua colonización-El Burgar, Paret Delgada-

Teniendo en cuenta los antecedentes expuestos y las características de las áreas donde se ubicaron los de fundación posterior, pueden extraerse una serie de consecuencias de orden histórico que caen fuera del ámbito de este estudio.

\section{Situación y emplazamiento}

Observando el cuadro de altitudes (cuadro 1), se aprecia que la ubicación de los santuarios es muy variada, desde los que se hallan prácticamente a nivel del mar -Berà, $20 \mathrm{~m}$-, hasta los que se encuentran en las cotas más altas del territorio en cuestión -L'Abellera, 1020 $\mathrm{m}$. Pero más interesante que la altura absoluta sobre el nivel del mar, es la situación respecto de los accidentes del relieve. En este sentido, se aprecian varias constantes dignas de ser tenidas en cuenta.

El sector Ilano del Camp de Tarragona es el territorio que ha gozado tradicionalmente de un mayor desarrollo socioeconómico; aquí se ubican un total de once centros marianos- el 31 por ciento del total diocesano-; algunos están separados por escasa distancia y en un caso existen dos en el mismo núcleo de población -Misericòrdia y El Roser en Reus-

Las montañas del sector occidental son las que cuentan con el mayor número absoluto de eremitorios, trece en total, lo que representa el 36 por ciento en cifras relativas. En el grupo se incluyen los que se hallan situados en la banda de contacto entre el sector y las Arxiu d'Etnografia de Catalunya, $n^{\circ} 2,1983,22-45$ ISSN: 0212-0372. EISSN: 2014-3885 tierras que lo limitan; se trata de los santuarios de Montgoi, La Serra, La Jonquera y La Consolació.

Otra área en la que se concentran cuatro santuarios a escasa distancia es el reborde montañoso que separa la Conca de Barberà de las comarcas Ilanas de la Depresión Central catalana: EI Tallat, EI Claustre, Passanant y La Bovera.

La excepción en el gran vacío del sector NE lo constituye la ermita de Valldossera, cuya importancia viene dada por el desplazamien. to de población desde las alturas de Montagut hacia los valles que li. mitan el antiguo municipio por el Este.

Otro grupo lo constituyen una serie de santuarios situados a lo largo de la costa. Berà es el más oriental y El Camí de Cambrils el más meridional.

En el conjunto de centros, es digno de resaltar que algunos gozan de una polivalencia en cuanto a su situación. El ejemplo más representativo lo constituye el de La Roca, que está en el limite sur del Camp de Tarragona, en su sector llano, al tiempo que en el limite montañoso; por su proximidad al mar y su elevación, puede considerarse como enclavado en el sector costero, de cuyas características participa.

En cuanto al emplazamiento, las características del lugar concreto en que se encuentra cada santuario, sus relaciones con la topografía local, es un aspecto de suma importancia y por tanto digno de estudio. A este respecto, hay que convenir en que los santuarios marianos estudiados ocupan lugares privilegiados, dotados de ventajas de diversa indole; unos están en lugares elevados, de gran dominio panorámico; otros en abrigos naturales, donde el microclima local queda suavizado en sus valores más extremos. Las condiciones del emplazamiento son variadas, pero en los treinta y seis centros estu. diados se observan unas constantes que permiten establecer una cierta sistematización.

Un gran número de centros están construidos en lugares elevados respecto del terreno circundante, por lo que el campo de su domien los que se muy extenso. Esta circunstancia se hace muy patente ubicació se hallan situados en el sector llano, que buscan para su sentativo el cerro aislado, la elevación natural. Los casos más representativos son los siguientes: 
- Berá, situado en un promontorio rocoso que se eleva en la vertical de la costa.

- Montornès. En un cerro a poniente del núcleo de población.

- El Cami. En las mismas condiciones, dominando la panorámica que se extiende hasta la línea de la costa.

- La Roca. Al Oeste de Montroig, en la estribación de una mole rocosa dominando todo el sector suroeste del Baix Camp.

- Loreto de Bràfim. En una elevación desde la que se extiende un panorama dilatado por las tierras de cultivo en un amplio sector del Alt Camp, y hasta la sierra de Montferri en la margen izquierda del Gaià.

- El Lledó de Valls. En una elevación al Norte del núcleo de población.

El Roser de Vilallonga. En un altozano que domina las tierras adyacentes al Francoli en una amplitud considerable.

- La Serra. Se encuentra en una elevación extramuros de Montblanc.

- Montgoi. Asimismo en la cima de un cerro dominante sobre Vilaverd y su valle.

- Loreto de Ulldemolins. En su emplazamiento original, sobre un cerro de $774 \mathrm{~m}$. de altura, era visible desde la totalidad del valle.

Los santuarios del sector montañoso ven acentuada esta carac. teristica, puesto que a la ventaja de encontrarse en una cota elevada, están situados en lugares que constituyen por si mismos una atalaya natural, lo que les permite disponer de una panorámica muy extensa. Unos se encuentran en el borde de un sistema montañoso, encima de un escarpe; otros en la elevación más sobresaliente del entorno. En este grupo se incluyen los siguientes:

- Les Virtuts. En las estribaciones de la parte oriental del macizo de Prades, a 780 metros de altura, sobre el valle del río Glorieta, extendiendo su campo visual además, a buena parte del Camp de Tarragona.

- L'Abellera. Constituye la cota máxima del conjunto objeto de estudio. A 1020 metros de altura, está situada asimismo en una hendidura de la roca, sobre el valle alto del Brugent, siendo visible además desde amplios sectores de los altiplanos de Prades.

- Montsant. La situación es también al borde de un escarpe de paredes verticales que se desploman sobre el valle de Ulldemolins; domina todo el sector septentrional del Priorat y una parte de la comarca adyacente de Les Garrigues.

- Siurana. También en el sector superior del Priorat. Su situación es la más clara del grupo. El emplazamiento consiste en un plataforma elevada sobre el curso alto del río Siurana y en un aislamiento casi total. El desnivel es considerable y el escarpe sobre el que se asientan la iglesia y la población, presenta una elevación superior a los 250 metros.

- La Roca. Aunque su altura sea relativamente de poca consideración, su situación permite la observación directa del sur del Baix Camp.

- La Consolació. La ermita se encuentra en la cima de un cerro que se eleva sobre las tierras circundantes en la confluencia de los rios Siurana y Montsant. Son visibles la práctica totalidad de las tierras del Priorat, exceptuando el sector norte; debido a esta circunstancia, se la conoce popularmente en la comarca como el "balcón del Prioratn.

- Puigcerver. El monte que lleva el nombre de la ermita alcanza una altura de 831 metros; no existe ninguno en una extensa área que le supere. Desde la cima se extiende el campo visual por la mayor par. te del Priorat, hasta el Montsant, las estribaciones meridionales de la Sierra de Prades y más de la mitad del llano del Camp de Tarragona, hasta el limite oriental, así como una gran extensión marítima. El san. tuario se encuentra a unos 400 metros de la cima hacia Levante y en una cota ligeramente inferior.

- El Tallat. En una elevación sobre el borde montañoso al Norte de la Conca de Barberà. Su situación permite contemplar la práctica totalidad de esta comarca, además de puntos más lejanos, como e Montsant, macizo de Prades, parte de Les Garrigues, Urgell y Segarra. Su situación, junto con Puigcerver, es la más privilegiada en este sentido.

- Passanant. En el mismo borde montañoso que El Tallat, su situación es idónea para la observación de extensos territorios de la parte septentrional.

- La Bovera. En un cerro, sobre el valle del río Corb. Su situación y altura le permiten destacarse sobre las tierras circundantes, especialmente las comarcas de Urgell y Segarra.

De la conjunción entre la situación espacial y el emplazamiento de la mayor parte de los centros estudiados, se desprende que es po- 


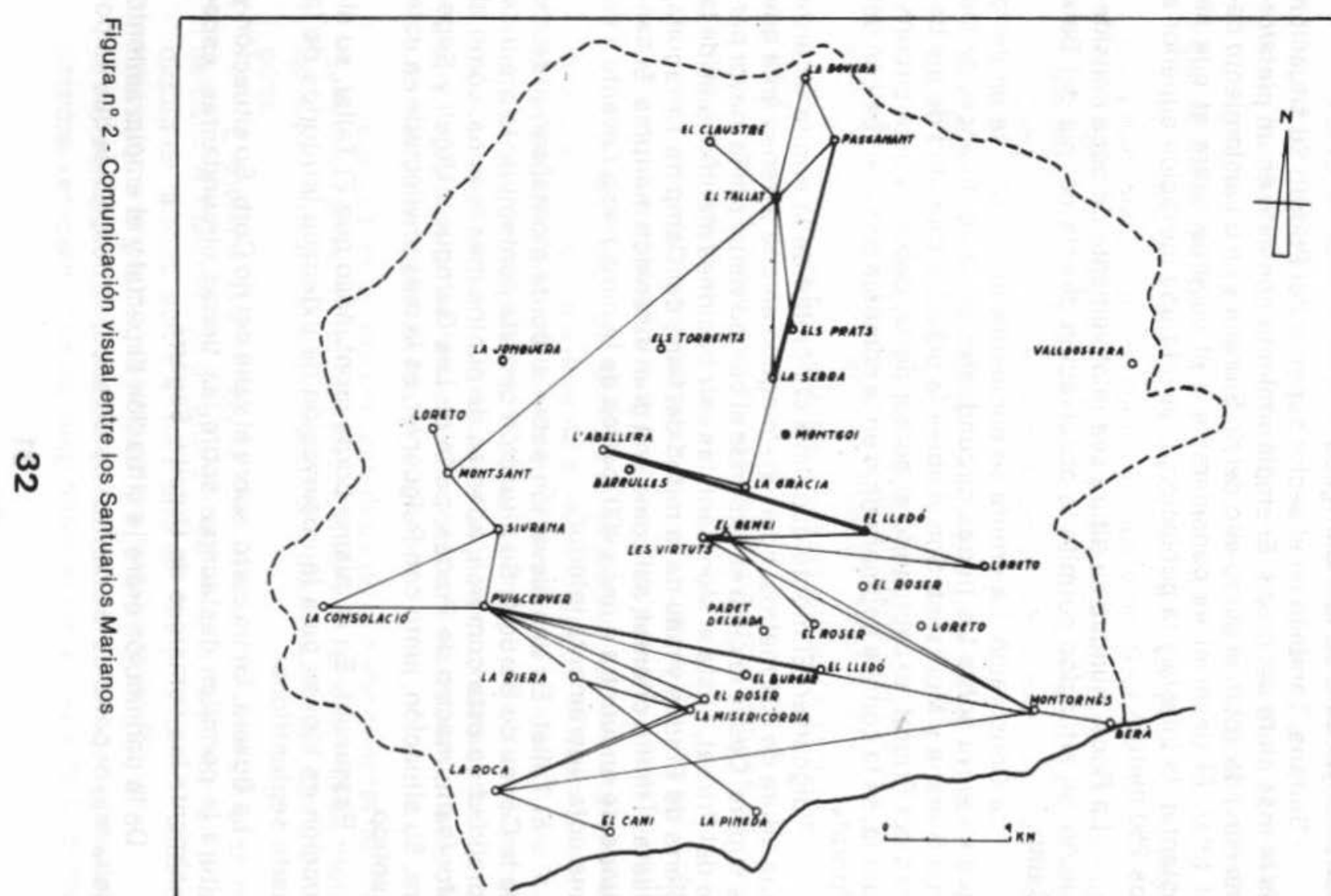

Arxiu d'Etnografia de Catalunya, $n^{\circ} 2,1983,22-45$

ISSN: 0212-0372. EISSN: 2014-3885

http://antropologia.urv.cat/revistarxiu 
sible la observación directa de la mayor parte del territorio de la diócesis desde alguno de ellos. Por otra parte, salvo escasísimas excepciones -Valldossera, Montgoi, Barrulles-, la comunicación visual entre los mismos es posible a través de una tupida red que cubre casi la totalidad del espacio de la diócesis (figura 2).

Contrastando con estos santuarios, existe un grupo cuyo emplazamiento tiene unas condiciones de signo contrario: se encuentran en lugares recónditos, en el fondo de un valle, junto a las riberas abruptas de un curso de agua, etc. En este caso están El Remei, Valldossera, Loreto de Renau, El Claustre, Els Torrents y Barrulles. Las razones para su localización y emplazamiento son distintas para cada uno de ellos.

\section{Vias de comunicación}

Para un gran número de santuarios, el emplazamiento es inmediato a una vía de comunicación que, en unos casos ha perdido importancia con la construcción de la red actual y en otros la conservan todavia. Los casos más patentes figuran en el cuadro 2.

Cuadro $n^{\circ}$ 2.- Situación de los santuarios marianos de la diócesis de Tarragona en relación con las vias de comunicación antiguas.

Santuario

El Tallat

El Lledó

(Pobla M.)

El Cami

Els Prats $(5)$
Camino

Guimerà-Senant Montblanc-Vallbona de Barberà

Tarragona-Montblanc Tarragonès-Alt Camp- Conca de (Via Aurelia)

Tarragona-Tortosa (via Augusta o Máxima)Ebro Montblanc-Tallat- Conca de Barberà-Urgell

Senant Montblanc-

Passanant-Guimerà

La Bovera
Radio acción del camino Barberà-Lleida

Camp de Tarragona y tierras del
Camino real Verdú- Urgell-Conca de Barberà Guimerà-Conca de Barberà

1




\begin{tabular}{|c|c|c|}
\hline Passanant & $\begin{array}{l}\text { Guimerà-Belltall- } \\
\text { El Tallat-Senant }\end{array}$ & $\begin{array}{l}\text { Urgell-Conca de Barberà } \\
\text { Originariamente unian los casti- } \\
\text { llos de Guimerà, Ciutadilla, Bell- } \\
\text { tall y el santuario de El Tallat } \\
\end{array}$ \\
\hline El Claustre & El Tallat-Maldà & Conca de Barberà-Urgell \\
\hline Montgoi & $\begin{array}{l}\text { Tarragona-Lleida } \\
\text { (Via Aurelia) }\end{array}$ & $\begin{array}{l}\text { Camp de Tarragona-Depresión } \\
\text { Central }\end{array}$ \\
\hline $\begin{array}{l}\text { El Lledó } \\
\text { (Valls) }\end{array}$ & Valls-Cabra & $\begin{array}{l}\text { Alt Camp-Conca de Barberà- } \\
\text { La Segarra }\end{array}$ \\
\hline Paret Delgada & Vilallonga-la Selva & Sector central Camp de Tarragona \\
\hline Els Torrents & $\begin{array}{l}\text { Poblet-Vimbodi- } \\
\text { Lleida }\end{array}$ & Poblet-Garrigues-Segrià \\
\hline $\begin{array}{l}\text { El Roser } \\
\text { (Vallmoll) }\end{array}$ & Vallmoll-Valls & Alt Camp \\
\hline $\begin{array}{l}\text { El Roser } \\
\text { (Vilallonga) }\end{array}$ & \begin{tabular}{|l|l}
$\begin{array}{l}\text { Tarragona-Lleida } \\
\text { (Via Aurelia) }\end{array}$ & \\
\end{tabular} & $\begin{array}{l}\text { Camp de Tarragona-Depresión } \\
\text { Central }\end{array}$ \\
\hline $\begin{array}{l}\text { El Roser } \\
\text { (Reus) }\end{array}$ & \begin{tabular}{|l|l|}
$\begin{array}{l}\text { Reus-La Selva-Valle } \\
\text { del Francoli }\end{array}$ \\
\end{tabular} & $\begin{array}{l}\text { Sector norte del Baix Camp } \\
\text { Depresión Central }\end{array}$ \\
\hline Misericòrdia & Reus-Cambrils & Sector sur del Baix Camp \\
\hline La Gràcia & \begin{tabular}{|l|} 
Alcover-Mont-ral \\
\end{tabular} & Alcover-Valle del Brugent \\
\hline $\begin{array}{l}\text { Loreto } \\
\text { (Bràfim) }\end{array}$ & Valls-Bràfim-Rodonyà & $\begin{array}{l}\text { Enlace con el camino Montblanc- } \\
\text { Vilafranca por el collado de } \\
\text { Sta. Cristina }\end{array}$ \\
\hline Berà & Via Augusta o Máxima & Penedès-Tarragonès \\
\hline Valldossera & \begin{tabular}{|l|} 
Santes Creus- \\
Pontons-Vilafranca \\
Santa Coloma-Querol \\
Plà de Manlleu \\
\end{tabular} & $\begin{array}{l}\text { Valle del Gaià-Penedès } \\
\text { Conca de Barberà-Penedès }\end{array}$ \\
\hline $\begin{array}{l}\text { Loreto } \\
\text { (Ulldemolins) }\end{array}$ & \begin{tabular}{|l|}
$\begin{array}{l}\text { Camino real Lleida- } \\
\text { Reus }\end{array}$ \\
\end{tabular} & $\begin{array}{l}\text { Priorat, Garrigues, Lleida, Ribera } \\
\text { Ebre }\end{array}$ \\
\hline La Serra & Via Aurelia & $\begin{array}{l}\text { Camp de Tarragona-Depresión } \\
\text { Central }\end{array}$ \\
\hline
\end{tabular}

El resto de los santuarios, o bien se encuentran en un aislamiento más o menos riguroso, o los caminos que pasan por las inmedia-

ciones poseen una escasa importancia como vias de paso con un interés puramente local, aparte de servir de acceso al propio santuario (figura 3).

\section{Climatología}

En la elección del emplazamiento de los centros estudiados, el factor clima ocupa un lugar secundario, privando de forma clara otros condicionantes. Sólo dos casos están situados a propósito para preservarles de los rigores climatológicos procedentes del Norte o el Oeste; se trata de L'Abellera, encajada en una balma y orientada hacia el Sur, y El Remei, en un recodo del río Glorieta abierto hacia el Este y protegido también por altos escarpes. El resto de santuarios se encuentran en lugares donde la influencia de las corrientes frias es bien patente. El hecho es tanto más significativo cuanto que en la mayoria de los casos bastaba con variar emplazamiento en muy escasos metros para disponer de unas condiciones climatológicas más favorables.

Sin embargo, al plantearse la orientación del edificio, se buscó por lo general mitigar los rigores climáticos, lo que se consigue mediante la exposición hacia el Este o el Sur. Unicamente tres santuarios están orientados hacia el Norte -Siurana, Loreto de Renau y Berà-; en el resto, la entrada o la fachada principal están orientadas hacia el Sur en su mayoria, o hacia el Este, presentando escasisimas aberturas en la fachada norte. Algunos presentan espacios porticados orientados hacia el mediodia, reforzando así la protección res. pecto de los rigores climáticos: Puigcerver, El Camí, La Bovera, La Roca, Misericòrdia, La Pineda y Berà.

\section{Hidrografia}

Un grupo de santuarios se encuentra situado junto a corrientes hidrográficas, ya sean continuas o intermitentes. Son los siguientes:

- La Riera, junto a la riera de Alforja.

- Paret Delgada, en la riera de La Selva.

- El Roser de Vallmoll, en el barranco Fonollosa.

- Loreto de Bràfim, a escasa distancia del rio Gaià.

- La Serra, cerca del Francolí.

- La Consolació, a caballo entre los ríos Siurana y Montsant.

- El Remei, junto al río Glorieta.

- Els Torrents, a corta distancia del Francolí. 


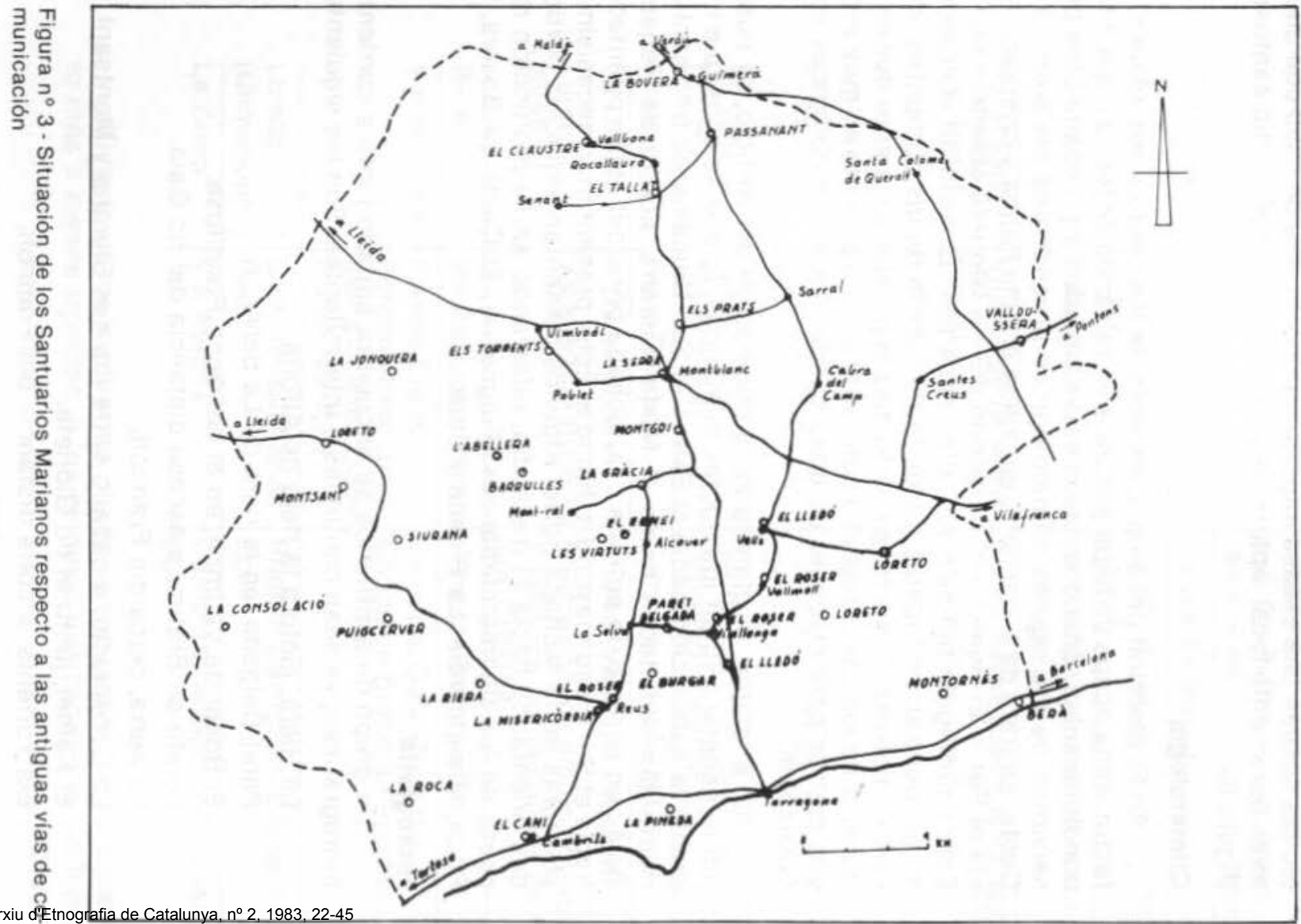

Arxiu d'Etnografia de Catalunya, $n^{\circ} 2,1983,22-45$

ISSN: 0212-0372. EISSN: 2014-3885

http://antropologia.urv.cat/revistarxiu 
- El Lledó de la Pobla de Mafumet, en su emplazamiento original junto al Francoli.

- Loreto de Renau, junto al torrente de Renau.

- Valldossera, junto al barranco de Ballestà.

Sin embargo, la escasez de la red hidrográfica, el exiguo caudal de los ríos y el hecho de que la mayor parte de las corrientes tengan carácter intermitente, resta una gran significación a este capítulo. En ningún caso la existencia de una corriente hídrica en las inmediaciones del santuario influye de una forma patente en la vida del mismo.

En cambio, son abundantes los casos en que el eremitorio se sitúa en las proximidades de una fuente de caudal más o menos abundante. En este caso se encuentran El Remei, La Gràcia, Els Torrents, La Jonquera, El Montsant, L'Abellera, Barrulles, La Roca, El Roser de Vallmoll, Loreto de Renau y El Claustre.

Algunas de estas fuentes gozan de propiedades medicinales, como las que existen en las proximidades de EI Tallat o El Claustre. EI valle en el que se sitúan estos dos santuarios es rico en aguas mineromedicinales; algunos manantiales son objeto de explotación comercial en la actualidad y dieron origen a la existencia hasta época reciente de establecimientos hoteleros que aprovechaban las propiedades de estas aguas. El área se extiende por las inmediaciones de las ermitas de la Bovera y Passanant y en general por todo el rio Corb y barrancos tributarios; a escasa distancia de La Bovera existe en explotación todavia el balneario de Vallfogona de Riucorb.

Los santuarios emplazados en lugares carentes de corrientes hidricas o manantiales solucionan el problema de aprovisionamiento a base de recogida de las aguas pluviales con sistemas de canalización de las cubiertas y cisternas de almacenamiento; ello permitia cubrir las necesidades de consumo humano e incluso el cultivo de pequeñas parcelas -por ej. La Bovera-. En otros casos la solución se arbitró a base de pozos, por ejemplo Paret Delgada, Valldossera, Roser de Vilallonga.

\section{RASGOS ECONÓMICOS}

Uno de los aspectos a tener en cuenta en el estudio que nos ocupa, es la relación entre la situación de las ermitas con el entorno so. cioeconómico en el que están insertas. Existen en este sentido varios grupos bien diferenciados. 
En primer lugar, varios santuarios se erigieron en un contexto de econóa urbana, donde a la actividad agraria se sumaban otras de tipo artesanal, comercial o de servicios. Tal es el caso de La Misericòrdia y El Roser en Reus, El Lledó en Valls y La Serra en Montblanc. a existencia de estos santuarios estuvo favorecida por el tipo de ac tividades que se desarrollaba en su área de influencia, donde el predominio de población urbana era manifiesto.

En el caso de La Serra, en la época de su fundación -finales del siglo XII- las actividades de la población se veian impulsadas por la circunstancia de figurar Montblanc en la ruta que unia el Mediterráneo con las tierras del interior a través del camino real TarragonaLleida.

Los santuarios de Valls y Reus son de época más tardia -finales del siglo XIV y principios del XV respectivamente-, cuando ya las ciudades habian adquirido una significación económica notable.

Un segundo grupo puede establecerse con los que se encuentra en un territorio eminentemente agricola, con exploconstruc taciones que ya eran intensivas ción; ejemplos de este grupo son los de El Burgar, Paret Delgada, Roser de Vilallonga y El Lledó de Pobla de Mafumet. Otra trándose en áreas de cultivos de secano, presentan las mismas ca racterísticas de cultivo intensivo; en el sector viticola del Priorat está La Consolació; en la Conca de Barberà se combinan la vid con los ce reales, mientras que en el Camp de Tarragona predomina fundamen. talmente la Ilamada trilogia mediterránea: cereales, vid y olivo.

Otro grupo, en fin, se establece con una serie de centros ubica. dos en lugares donde la actividad agricola es secundaria, siendo predominante la riqueza forestal. En Puigcerver fue importante la exploqa la elaboración de carbón vegetal, actividad de gran incidencia económica en épocas pasadas. En L'Albellera Barrulles, a la explotación forestal de la encina y el pino hay que

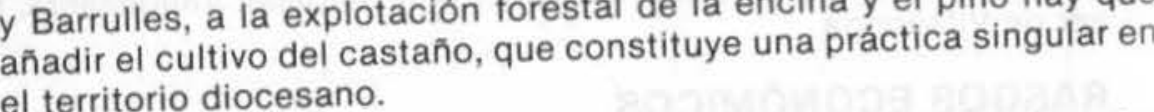

La ganaderia es objeto de una notable dedicación en el sector ce Barberà, donde existe importante cabaña ovina, que es de suponer lo fue mucho más en la época de fundación de los santuarios alli existentes. El mismo nombre de Els Prats es in. dicativo de la dedicán intensiva a esta actividad. No hay que desArxiu d'Etnografia de Catalunya, $n^{\circ} 2,1983,22-45$ cartar, asimismo, la cria de ganado en el Camp de Tarragona en los terrenos dedicados a los cultivos de secano.

Hay que tener en cuenta, no obstante, que las condiciones económicas actuales difieren sensiblemente de las existentes en la época en que fueron construidos la mayor parte de los centros estudiados; la regresión de algunos cultivos y la introducción de otros, junto con las transformaciones sociales, implican que la actividad agraria tenga un signo distinto del que tuvo en la época histórica de la funda ción de los santuarios objeto de estudio. Por otra parte, la despobla ción rural en unos casos y el diferente signo económico en otros han cambiado el entorno socioeconómico, provocando el auge en algu nos santuarios -p. ej. Misericòrdia, Berà, La Serra, Lledó de Valls- y e abandono o semiabandono en otros -El Tallat, Les Virtuts, E Montsant-

En algunos casos, el establecimiento de un santuario está relacionado de forma muy directa con la repoblación y el auge económi. co del sector. En este caso está. La Jonquera, que funcionó desde sus inicios como una granja perteneciente al monasterio de Poblet, que estimuló de forma muy activa la repoblación del territorio. Otro ejemplo en este sentido es El Claustre, que a consecuencia de ciertas dis posiciones del Concilio de Trento, procedió a repoblar intensamente el valle en el que se encuentra ubicado el santuario y a través de ce siones de tierras dio origen al pueblo actual de Vallbona de les Monges.

\section{Economia marginal}

Las actividades económicas marginales -caza, recolección- tu vieron una importancia muy significativa en épocas pasadas y a ellas no son ajenas los santuarios estudiados. En las proximidades de mu chos de ellos existió y existe todavia abundancia de recursos que, si bien en la actualidad tienen la consideración de deporte o actividad de pequef́o o nulo valor lucrativo, en la época histórica en que fueron construidos tenian un signo muy distinto. La caza constituyó hasta época reciente un aporte alimenticio fundamental para amplios sec tores de la población; en cuanto a la recolección, especialmente en la parte montañosa, algunas especies son todavia una fuente de ingre sos para muchos de sus habitantes, aunque de signo complementario no por ello despreciable.

La caza es abundante en la práctica totalidad de los santuarios estudiados; la existencia de masas boscosas considerables en la 
época en que fueron construídos, hace pensar que lo fue mucho más que en la actualidad.

En las proximidades de todos los santuarios del sector montañoso abunda la caza mayor, particularmente la especie del jabalí, propiciada por la existencia de grandes masas de encinas o castaños, cu. yos frutos constituyen la base de su alimentación. Abunda también la caza menor, aunque ésta es más significativa en las áreas Ilanas. Si bien en la actualidad está muy diezmada, hay que pensar que en épocas anteriores su abundancia fue mucho mayor. Todavia hoy la abundancia de algunas especies determinadas -jabalies en la montaña y conejos al Norte de la diócesis-son causa de problemas por sus daños a los cultivos.

La recolección de plantas medicinales y aromáticas es una actividad que conserva todavia cierta importancia económica en algunos sectores de población; una gran parte de las ermitas estúdiadas dispone en su entorno inmediato de abundantes recursos de este tipo. Se ha constatado la existencia de especies aprovechables en la práctica totalidad de los santuarios, constituyendo una excepción aquellos en los que el territorio se ha dedicado por completo a usos agricolas. Abundan especialmente el tomillo (Thymus vulgaris), romero (Rosmarinus officinalis), espliego (Lavandula officinalis), salvia (Salvia officinalis), abrótano (Artemisia abrotanum), etc. en una relación que se haria muy extensa y que ha pasado al acervo de la medicina popular.

En cuanto a las setas, los lugares más privilegiados son los co. rrespondientes a las montañas de Prades y de Montagut. Existe abundancia y variedad de especies en las proximidades de Siurana, Les Virtuts, La Gràcia, L'Abellera, Barrulles, El Remei, Valldossera y Montsant. Se recolectan especialmente el nizcalo o "rovelló" (Lactarius deliciosus), oronja o "reig" (Amanita caesaria), friolero o "fredolicn (Tricholoma terreum), pebrazo o upebràs" (Lactarius piperatus) y otras especies menos apreciadas. La recolección de algunas especies ha constituido tradicionalmente una fuente de ingresos para muchos habitantes de estos lugares.

\section{INTEGRACIÓN ESPACIAL}

\section{Relaciones con el hábitat}

Por la misma naturaleza de los centros estudiados y teniendo en cuenta que cada uno de ellos está inmerso en un área de influencia, es interesante considerar de qué forma los santuarios marianos se integran en el entorno y qué relaciones se establecen entre ellos y los núcleos de población a los que administrativamente pertenecen.

En principio, pueden establecerse dos categorias entre los santuarios estudiados: Ios que se insertan en el ambiente socioeconómico del sector, formando parte del hábitat en el que se hallan y los que se encuentran en un aislamiento más o menos destacado.

Un total de once eremitorios se encuentran en pleno núcleo de población, ya sea porque fueron ubicados alli desde el mismo origen -EI Cami, Siurana, Passanant, El Lledó de La Pobla de Mafumet, Loreto de Ulidemolins, El Roser de Vallmoll y Montgoi-, o bien porque la expansión urbana les envolvió hasta quedar inmersos en el mismo núcleo, como son los casos de La Misericórdia y Roser en Reus, Lledó en Valls y La Serra en Montblanc.

En otros casos, el núcleo de población surgió por la existencia previa del santuario y el poblamiento se debió a la influencia del mismo. En esta situación están La Jonquera de La Pobla de Cérvoles y EI Claustre de Vallbona de les Monges, ya comentados.

Otros se encuentran inmersos en zonas de cultivo formando parte del entorno, con el que están plenamente identificados. Son todos los restantes del Camp de Tarragona; situados a distancia variable de los núcleos de población, participan plenamente de la organización social del sector, tanto si se trata de población concentrada - La Riera, Loreto de Bráfim, Montornès, Loreto de Renau, Els Prats, La Con. solació, Barrulles- como dispersa -El Burgar, La Gràcia, Valldossera-

Existen en último lugar, una serie de santuarios ubicados en lugares de un aislamiento más o menos extremo; en algunos, la accesibilidad ha sido tradicionalmente difícil y lo sigue siendo en la actualidad. Asimismo, la distancia al núcleo de población a cuya jurisdic. ción pertenecen es grande. Entre ésto conviene citar como casos destacados, Puigcerver, Montsant, L'Abellera y Les Virtuts. En algunos casos los accesos son dificiles por la topografia del terreno -Montsant-o por el estado de abandono del santuario -Les Virtuts-; en cambio, en otros se han trazado nuevas vias de acceso o se han mejo. rado las existentes, que permiten el paso a los modernos medios de locomoción -Puigcerver, La Consolació, La Bovera, La Roca, L'Abellera-. 


\section{Limites administrativos}

La situación de los santuarios estudiados en el contexto de la división administrativa del territorio, ofrece unas constantes dignas de resaltar. Independientemente de otras consideraciones de orden histórico, el hecho es que se observa una coincidencia en la situación de muchos santuarios en los límites administrativos del obispado, la parroquia o el municipio. Como ha señalado J. Prat, ésta es una caracteristica común a la mayor parte de los santuarios marianos de Catalunya. Los casos más patentes figuran en el cuadro 3.

Cuadro $n^{\circ}$ 3.- Situación liminar de los santuarios marianos de la diócesis de Tarragona.

\begin{tabular}{|c|c|c|c|}
\hline \multirow[b]{2}{*}{ Santuario } & \multirow[b]{2}{*}{ Diocesanos } & \multicolumn{2}{|l|}{ Limites } \\
\hline & & Comarcales & Municipales \\
\hline Berà & $\begin{array}{l}\text { Tarragona-Barce- } \\
\text { Iona (Div. antigua) }\end{array}$ & $\begin{array}{l}\text { Tarragonès - } \\
\text { Baix Penedès }\end{array}$ & $\begin{array}{l}\text { Roda de Berà - } \\
\text { S.Vicente de Cal- } \\
\text { ders }\end{array}$ \\
\hline Valldossera & $\begin{array}{l}\text { Tarragona-Barcelo- } \\
\text { na-Vic }\end{array}$ & $\begin{array}{l}\text { Alt Camp - Alt } \\
\text { Penedès }\end{array}$ & $\begin{array}{l}\text { Querol (Montagut) } \\
\text { Pontons }\end{array}$ \\
\hline La Bovera & \begin{tabular}{|l|} 
Tarragona - \\
Solsona
\end{tabular} & $\begin{array}{l}\text { Conca Barberà - } \\
\text { Urgell }\end{array}$ & $\begin{array}{l}\text { Guimerà - Verdú - } \\
\text { Ciutadilla }\end{array}$ \\
\hline Passanant & $\begin{array}{l}\text { Tarragona - } \\
\text { Solsona }\end{array}$ & $\begin{array}{l}\text { Conca Barberà - } \\
\text { Urgell }\end{array}$ & \\
\hline El Claustre & $\begin{array}{l}\text { Tarragona - } \\
\text { Solsona }\end{array}$ & $\begin{array}{l}\text { Conca Barberà - } \\
\text { Urgell }\end{array}$ & $\begin{array}{l}\text { Vallbona de les } \\
\text { Monges - Rocafort } \\
\text { de Vallbona }\end{array}$ \\
\hline El Tallat & & $\begin{array}{l}\text { Conca Barberà } \\
\text { Urgell }\end{array}$ & $\begin{array}{l}\text { Rocallaura-Blanca } \\
\text { fort - Vallbona de } \\
\text { les Monges - Es- } \\
\text { pluga de F. }\end{array}$ \\
\hline La Jonquera & Tarragona - Lleida & $\begin{array}{l}\text { Conca Barberà } \\
\text {-Garrigues }\end{array}$ & \\
\hline $\begin{array}{l}\text { Loreto } \\
\text { (Ulldem.) }\end{array}$ & $\begin{array}{l}\text { Tarragona - Lleida } \\
\text { Tortosa }\end{array}$ & \begin{tabular}{|l} 
Priorat - Garrigue \\
Conca de Barbe
\end{tabular} & \\
\hline
\end{tabular}

Arxiu d'Etnografia de Catalunya, nº 2, 1983, 22-45 ISSN: 0212-0372. EISSN: 2014-3885
http://antropologia.urv.cat/revistarxiu

\begin{tabular}{|c|c|c|c|}
\hline Montsant & & & $\begin{array}{l}\text { La Morera de } \\
\text { Montsant - Cornu- } \\
\text { della-Ulldemolins } \\
\end{array}$ \\
\hline Barrulles & & $\begin{array}{l}\text { Baix Camp - Alt } \\
\text { Camp }\end{array}$ & $\begin{array}{l}\text { Capafons - Mont- } \\
\text { ral-Prades } \\
\end{array}$ \\
\hline La Gràcia & & & \begin{tabular}{|l}
$\begin{array}{l}\text { Alcover - Mont-ral } \\
\text {-La Riba }\end{array}$ \\
\end{tabular} \\
\hline El Remei & & & Alcover - Mont-ral \\
\hline Les Virtuts & & $\begin{array}{l}\text { Baix Camp - Alt } \\
\text { Camp }\end{array}$ & $\begin{array}{l}\text { Albiol - Alcover - } \\
\text { Mont-ral }\end{array}$ \\
\hline $\begin{array}{l}\text { Loreto } \\
\text { (Bráfim) }\end{array}$ & & te & $\begin{array}{l}\text { Bràfim - Montferri } \\
\text { Vilabella }\end{array}$ \\
\hline \begin{tabular}{l|l} 
Loreto \\
(Renau)
\end{tabular} & & $\begin{array}{l}\text { Tarragonès - Alt } \\
\text { Camp }\end{array}$ & \\
\hline $\begin{array}{l}\text { El LLedó } \\
\text { (Pobla de M) }\end{array}$ & & & $\begin{array}{l}\text { La Pobla de Mafu- } \\
\text { met - Contantí }\end{array}$ \\
\hline \begin{tabular}{l|} 
El Roser \\
(Vilallonga)
\end{tabular} & & \begin{tabular}{|l|} 
Tarragonès - Alt \\
Camp
\end{tabular} & \begin{tabular}{|l|} 
Vilallonga - \\
Rourell \\
\end{tabular} \\
\hline $\begin{array}{l}\text { Paret } \\
\text { Delgada }\end{array}$ & & $\begin{array}{l}\text { Baix Camp - Tarra- } \\
\text { gones -Alt Camp }\end{array}$ & $\begin{array}{l}\text { La Selva del } \\
\text { Camp - Vilallonga } \\
\text { - Alcover } \\
\end{array}$ \\
\hline El Burgar & & & Reus - Contanti \\
\hline La Pineda & & & \begin{tabular}{|l} 
Vila-seca- \\
La Canonja \\
\end{tabular} \\
\hline La Roca & & Tarragona-Tortosa & $\begin{array}{l}\text { Montroig - Vilano- } \\
\text { va d'Escornalbou }\end{array}$ \\
\hline Puigcerver & & Baix Camp -Priorat & Alforja-Riudecols \\
\hline $\begin{array}{l}\text { La Consola- } \\
\text { ció }\end{array}$ & $\begin{array}{l}\text { Tarragona - } \\
\text { Tortosa }\end{array}$ & & $\begin{array}{l}\text { Gratallops - Torro- } \\
\text { ja } \\
\text { Vilella Alta }\end{array}$ \\
\hline
\end{tabular}

Además de los citados, es probable que existan otros casos que se situaron en los límites de parroquias o posesiones del arzobispado o de algún monasterio y que no se corresponden con las actuales debido a las modificaciones sufridas a partir del siglo pasado. 


\section{CONCLUSIONES}

El territorio objeto de estudio presenta unas caracteristicas físicas de una maracada diversidad en cuanto al relieve, hidrografia, economia, población, etc. Sin embargo, los santuarios marianos que se encuentran en el mismo, tiene unos puntos de coincidencia y unas constantes que pueden sintetizarse en los siguientes puntos:

- Son muy abundantes en el sector central del Camp de Tarragona. asi como en las montañas occidentales; en cambio hay un gran vacio en el Nordeste.

- Están situados en lugares preeminentes del terreno, pudiendo establecerse una red que permite la observación directa de la práctica totalidad del territorio. El sector costero dispone, asimismo, de centros distribuidos espacialmente a lo largo de su fachada maritima.

- Una gran parte están emplazados junto a vias de comunicación que gozaron en épocas pasadas de gran radio de influencia.

- Un grupo numeroso de santuarios dispone en las proximidades de una corriente hídrica o un manantial. En tres casos, el manantial es de aguas medicinales y se sitúa en el mismo santuario o sus inmediaciones.

- La existencia de fuentes económicas marginales -caza, recolección - es común en la práctica totalidad de los santuarios.

- Son cuantitativamente más numerosos los santuarios ubicados en centros de población o a escasa distancia, que los que se encuentran en lugares alejados de cualquier centro urbano.

- Prácticamente la mitad de los centros estudiados se encuentran en los límites territoriales del arzobispado o del término municipal al que pertenecen. En dos casos - La Pineda y Puigcerver- la divisoria pasa por el mismo Santuario. Estas situaciones han sido tradicionalmente causa de conflictos entre comunidades vecinas, que se arrogaban la supremacia en el dominio del santuario.
NOTAS:

(1) Se ha incluido la época de fundación, porque a pesar de tener un interés fundamentalmente histórico, es de sumo interés para establecer las condiciones fundamenmicas del sector cuando se erigieron, al tiempo que explican en parsiocono. ción espacial

(2) Probablemente existía ya a finales del siglo anterior, La localización primitiva de la imagen fue, según F. Blasi en la ermita de Sant Joan del Lledoner, en terreno de la diato al rio Francoli. Es muy probable que esta ermita sea la iner, entreno inme Concili, que después de tener jurisdicción propia, pasó a ser sufragánea de Saan de re del Codony y más tarde trasladada al núcleo de La Pobla sufragánea de Sant Pe manece la imagen desde trasladada al nucleo de La Pobla de Mafumet, donde per

(3) Según la tradición, existia ya un

(3) Según la tradición, existia ya un centro con esta advocación a mediados del siglo

(4) La imagen, según la tradición, existía ya en el siglo $X$.

\section{BIBLIOGRAFIA}

BLASI VALLESPINOSA, F.: Les riberes del Gaià, Barcelona, 1931 Id. id.: Santuaris marians de la provincia de Tarragona, Revista del
Centre de Lectura, Reus, 1933.

CARRERAS CANDI, F:: Geografia general de Catalunya, Marín, BarceIona, 1908.

CORTIELLA, F.: Noticies del Codony, (en "Universitas Tarraconensisn, tomo IV) Tarragona, 1982.

IGLESIES, J.: Les muntanyes de Prades, EI Montsant i Serra La Llena, Centre de Lectura, Reus, 1929.

IGLESIES, J. y J. SANTASUSAGNA: Del Camp de Tarragona a l'Ebre, Reus, 1931.

PLADEVALL, A. y F. CATALA ROCA: Els monestirs catalans, Destino, Barcelona, 1978.

PRAT, J:: Informe de la investigació sobre els santuaris marians a Catalunya, policopiada, Departament d'Antropologia, Universitat de Barcelona, Tarragona.

Id. id.: Els santuaris marians al Camp de Tarragona: algunes hipòtesis de treball, (en "Universitas Tarraconensis", tomo IV),
Tarragona, 1982.

SOLE Tarragona, 1982.

SOLE SABARIS, LI. y otros: Geografia de Catalunya, III t. Aedos, 1974 Barcelona, 1968-69: Catalunya visió, tomos I, IV, X, Taber,

Atlas històric, geogràfic i comarcal de Catalunya, Diàfora, Barcelona, 1978.

Mapa Telstar de Catalunya.

Mapa topográfico de España, escala 1:50000. 